\title{
Minimal Improvement in Height Deficit Post-Transplant
}

\author{
Richard N. Fine
}

Department of Pediatrics, State University of New York at Stony Brook, New York, USA

Infants, children and adolescents are frequently growth retarded at the time of renal transplantation. Catch-up growth post-transplant has traditionally been evaluated by improvement in the standard deviation scores (SDS) following successful transplantation. A more realistic method of assessment could be improvement in height deficit post-transplant.

The height deficit is defined as the number of centimeters below the 50th centile on the growth curve for chronologic age. Since infants, children and adolescents with growth retardation consequent to chronic renal disease and have the inherent potential for normal growth, it would seem appropriate to judge the impact of transplantation of growth in terms of the patients ability to achieve their inherent growth potential (50th centile for mid-parental height). Therefore, the magnitude of improvement in height deficit following transplantation was evaluated in the data from the North American Pediatric Renal Transplant Cooperative Study (NAPRTCS).

All patients registered with NAPRTCS prior to January 1, 1991 who were 3 to 16 years of age at the time of transplantation and were growth retarded with SDS below -2.0 were evaluated. The baseline and 2 years post-transplant mean SDS and height deficit were evaluated.

At baseline the mean SDS was $-3.44 \pm 0.05$ and height deficit was $-21 \pm 0.36 \mathrm{~cm}$. In the 524 recipients who had a functioning graft at 2 years, the change in SDS was $+0.32 \pm 0.04$ and in height deficit was $+0.75 \pm 0.23 \mathrm{~cm}$.

The shorter and younger recipients at baseline had a greater increase in the delta SDS. However, the change in height deficit was not dependent upon age, sex or magnitude of the height deficit at baseline.

Utilizing the above data a typical 5 year old recipient with an SDS of -3.00 and a height deficit of $20 \mathrm{~cm}$ at baseline would be expected to have a gain in height deficit at 2 years post- transplant of only $2.46 \mathrm{~cm}$ if a female, and $1.14 \mathrm{~cm}$ if a male. Therefore, less than $13 \%$ of the height deficit at baseline would be gained during the 2 year post-transplant period.

Significant height deficit is present in a significant number of infants, children and adolescents with chronic renal failure at the time of renal transplantation. Despite improvement in SDS following successful transplantation, "catch-up" growth as reflected in the change in height deficit is minimal. At 2 years post-transplant the change in height deficit can be anticipated to be less than $1.0 \mathrm{~cm}$.

Evaluating changes in height deficit rather than changes in SDS may be a more appropriate method of measuring the success of renal transplantation in improving the growth retardation resulting from chronic renal failure. 